\title{
Role of the polymer phase in the mechanics of nacre-like
}

\section{composites}

Tobias P. Niebel, Florian Bouville*, Dimitri Kokkinis, André R. Studart*

\section{Abstract}

Although strength and toughness are often mutually exclusive properties in manmade structural materials, nature is full of examples of composite materials that combine these properties in a remarkable way through sophisticated multiscale architectures. Understanding the contributions of the different constituents to the energy dissipating toughening mechanisms active in these natural materials is crucial for the development of strong artificial composites with a high resistance to fracture. Here, we systematically study the influence of the polymer properties on the mechanics of nacre-like composites containing an intermediate fraction of mineral phase (57 vol\%). To this end, we infiltrate ceramic scaffolds prepared by magnetically assisted slip 
casting (MASC) with monomers that are subsequently cured to yield three drastically different polymers: (i) poly(lauryl methacrylate) (PLMA), a soft and weak elastomer; (ii) poly(methyl methacrylate) (PMMA), a strong, stiff and brittle thermoplastic; and (iii) polyether urethane diacrylate-co-poly(2-hydroxyethyl methacrylate) (PUA-PHEMA), a tough polymer of intermediate strength and stiffness. By combining our experimental data with finite element modeling, we find that stiffer polymers can increase the strength of the composite by reducing stress concentrations in the inorganic scaffold. Moreover, infiltrating the scaffolds with tough polymers leads to composites with high crack initiation toughness $K_{I C}$. An organic phase with a minimum strength and toughness is also required to fully activate the mechanisms programmed within the ceramic structure for a rising R-curve behavior. Our results indicate that a high modulus of toughness is a key parameter for the selection of polymers leading to strong and tough bioinspired nacre-like composites.

\section{Keywords}

bio-inspired composites, fracture mechanisms, mechanical testing, stress concentrations, micro structures

\section{Introduction}

The increasing demand for strong, stiff and tough, yet light-weight materials for applications in transportation, energy harvesting and construction has led to extensive research into advanced structural materials. Unfortunately, the conflicting mechanisms underlying strength and toughness make these properties generally mutually exclusive in conventional engineering materials (Ritchie, 2011). Thus, a compromise is often required to find a suitable material for a specific load-bearing application. In many cases 
metals are chosen for their acceptable specific strength, stiffness and good workability, but may not fulfill special weight reduction requirements. In comparison, ceramics commonly exhibit a more favorable strength-to-weight ratio but are usually susceptible to brittle fracture. Bioinspired composite materials with a high volume fraction of ceramics and a compliant secondary phase are promising candidates to fill this gap (Ritchie, 2011; Wegst et al., 2015). In these composites, constituents are arranged in a highly sophisticated microstructure to achieve an unusual combination of low density, strength and high resistance against fracture (Bouville et al., 2014; Le Ferrand et al., 2015; Munch et al., 2008; Wegst et al., 2015).

One of the most studied natural archetypes for such materials is the nacreous part of sea shells, which reaches a remarkable strength and toughness due to a hierarchical brick-and-mortar structure containing 95 vol\% inorganic material (Espinosa et al., 2009; Meyers et al., 2008a; Sun and Bhushan, 2012). Bricks are formed by micron-sized slightly wavy aragonite $\left(\mathrm{CaCO}_{3}\right)$ platelets that are connected by mineral bridges and display nanoasperities on the surface (Barthelat et al., 2007; Evans et al., 2001; Wang et al., 2001). Thin layers of biopolymers - mainly chitin and different proteins - serve as nanometer-thick mortar between the platelets (Meyers et al., 2008a; Meyers et al., 2009). Such an intricate microstructure gives rise to several toughening mechanisms during fracture of nacre, including breaking of mineral bridges, friction and interlocking of rough platelets, crack deflection at the brick-mortar interface, platelet pull-out, and plastic deformation of the biopolymer (Barthelat et al., 2007; Evans et al., 2001; Katti et al., 2005; Song et al., 2003; Wang et al., 2001).

While several studies have been carried out to investigate the role of the platelet nanoasperities and waviness on the overall toughness (Barthelat et al., 2007; Evans et al., 2001; Katti et al., 2005; Wang et al., 2001), the contribution of the organic phase to 
the mechanical performance is less understood. This is explained by the small dimensions of the polymeric interlayers within nacre, which limit the possible tools that can be used to mechanically probe these materials. Important insights have been gained through in-situ mechanical testing using nanoindentation and atom force microscopy (AFM) (Meyers et al., 2009; Sarikaya, 1994). Nanoindentation revealed that the stiffness of the biopolymer layer is highly humidity dependent with an estimated elastic modulus as low as $100 \mathrm{~Pa}$ in the moist state and values on the order of GPa after drying (Meyers et al., 2009; Meyers et al., 2008b). Interestingly, this change in elasticity coincides with an overall embrittlement of nacre upon drying, which indicates the importance of the soft interlayer to facilitate tablet sliding during deformation (Barthelat et al., 2007). Organic ligaments between the inorganic platelets have been stretched and bent with an AFM-tip to demonstrate the dissipation of energy through domain unfolding in the polymer (Smith et al., 1999; Xu and Li, 2011). In addition to such in-situ mechanical testing, macroscopic experiments have been carried out on samples of the organic matrix obtained by demineralization of nacre (Bezares et al., 2008). Tensile tests on specimens prepared this way indicate that nacre's biopolymers exhibit strain-rate dependent strength and stiffness below $2 \mathrm{MPa}$ and $140 \mathrm{MPa}$, respectively (Dastjerdi et al., 2013; Lopez et al., 2014). In a study on nacre from three different species it was found that the highest interfacial toughness (crack direction parallel to long axis of platelet) correlates with the highest values of strength and elongation of the organic phase, which corresponds to the highest modulus of toughness (Dastjerdi et al., 2013).

While examining biological materials can provide valuable mechanistic insights and can benefit from the diversity of evolved species, this approach is limited to the structures and materials found in nature and does not allow for a systematic study of the isolated effect of the properties of the organic phase when varied within a wide design 
space (Dastjerdi et al., 2013; Rabiei et al., 2010). To complement experiments performed on natural structures, simulations of nacre-like virtual materials have been conducted using mainly finite element (FE) analysis. The importance of several toughening mechanisms arising from the architecture of the mineral phase has been confirmed through such analyses (Barthelat and Rabiei, 2011; Barthelat et al., 2007; Evans et al., 2001; Shao et al., 2012). However, numerical simulations to determine the properties of the organic phase remain ambiguous. Commonly, the calculated elastic modulus ranges from one to several GPa, which is much closer to the experimental value of the dried matrix than to its natural humid form (Askarinejad and Rahbar, 2015; Bezares et al., 2010; Katti and Katti, 2001; Xu et al., 2011).

Besides simulating natural nacre, a number of different models have been developed to identify the optimum design of artificial bioinspired brick-and-mortar composites (Barthelat, 2014; Barthelat et al., 2013; Begley et al., 2012; Pimenta and Robinson, 2014). These models often indicate that the failure mechanism is determined by the aspect ratio of the reinforcing tablets and the strength of the compliant interfaces. Depending on these parameters, the composite may fail due to platelet fracture or through matrix yielding and platelet pull-out. A multi-objective optimization scheme has recently revisited the ideal combinations of properties needed in a brick-and-mortar composite (Barthelat, 2014). According to this analysis, a brick-and-mortar structure is only beneficial at high brick volume fractions and if the interfaces are at least five times weaker than the utilized platelets. In contrast to other studies (Barthelat et al., 2013; Pimenta and Robinson, 2014), the overlap ratio between the platelets was suggested in this work to be less important than the parameters above (Barthelat, 2014).

A major drawback of analytical models is that they only consider a representative volume element of flat platelets. Thus, localized fracture phenomena and toughening 
mechanisms other than platelet pull-out cannot be captured (Barthelat, 2014; Begley et al., 2012). Structural features such as mineral bridges and nanoasperities for example are neglected in such analyses. Despite the valuable insights from theoretical models, it would therefore be highly desirable to construct biomimetic experimental models with deliberately varied polymer properties to gain a deeper understanding of the influence of the compliant matrix on the mechanics of a real nacre-like composite.

Although many pathways have been suggested to prepare artificial nacreinspired composites, only a few offer the possibility to vary the polymer properties without an impact on the mineral phase (Behr et al., 2015; Bonderer et al., 2008; Bouville et al., 2014; Deville et al., 2006; Ekiz et al., 2009; Erb et al., 2012; Libanori et al., 2013; Podsiadlo et al., 2007; Tang et al., 2003). Infiltrating porous ceramic scaffolds with an organic phase is a possible approach since the production of the two phases can be controlled separately. Methods to manufacture such scaffolds include ice-templating (Deville et al., 2006; Munch et al., 2008), spray-forming (Dwivedi et al., 2015) or magnetically assisted slip casting (MASC) (Le Ferrand et al., 2015). However, scaffolds have thus far only been infiltrated using a limited range of monomers and polymers, including for instance epoxy resins (Deville et al., 2006; Dwivedi et al., 2015) and poly(methyl methacrylate) (Launey et al., 2009; Le Ferrand et al., 2015; Munch et al., 2008; Naglieri et al., 2015).

In this paper, we study how the mechanical properties of the polymer matrix influence the overall behavior of scaffold-based nacre-inspired composites. Initially, scaffolds of 57 vol\% ceramic content with a nacre-inspired architecture are prepared from alumina microplatelets and silica nanospheres by MASC combined with hot pressing (Figure 1) (Le Ferrand et al., 2015). These scaffolds are infiltrated with (meth)acrylic monomers that are subsequently cured by radical polymerization to yield 
three polymers with drastically different properties: a soft and weak elastomer, a strong and stiff but brittle thermoplastic, and a tough polymer of medium strength and stiffness. The composites with the three different organic phases are compared by performing bending measurements, fracture toughness tests and electron microscopy. Finally, a FE model is developed to interpret the observed mechanical results and help set guidelines for the selection of polymers for the fabrication of tough nacre-like composites.

\section{Experimental procedure}

\subsection{Ceramic scaffold preparation}

Nacre-inspired ceramic scaffolds were prepared by MASC as described in the literature (Le Ferrand et al., 2015). The relative mineral content of the scaffold and the content of open porosity were determined by the Archimedes density method. Full details are also provided in the supporting information.

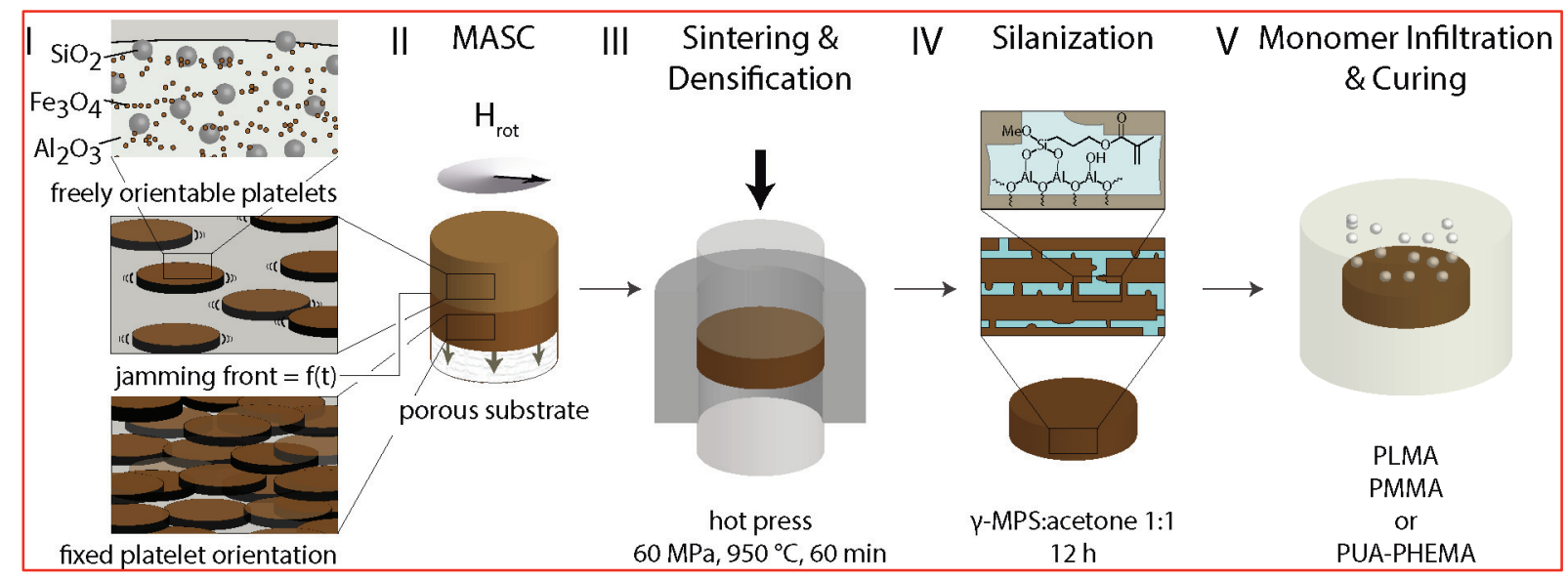

Figure 1: Procedure to prepare nacre-like ceramic-polymer composites. (I,II) Magnetically Assisted Slip Casting (MASC) and (III) consecutive sintering in a hot press to produce ceramic scaffolds is followed by (IV) silanization, (V) monomer infiltration and thermal curing to consolidate the polymer phase. $H_{r o t}$ indicates the rotating magnetic field applied during the MASC process. $\gamma$-MPS: 3-(trimethoxysilyl)propyl methacrylate, 
PLMA: poly(lauryl methacrylate), PMMA: poly(methyl methacrylate), PUA-PHEMA: polyether urethane diacrylate-co-poly(2-hydroxyethyl methacrylate).

Prior to the MASC process, silica nanospheres were electrostatically adsorbed on the surfaces of alumina microplatelets to introduce mineral bridges and nanoasperities after sintering (Figure 1, step I) (Le Ferrand et al., 2015; Libanori et al., 2016). Then, the platelets were rendered magnetically responsive by decorating their surface with superparamagnetic iron oxide nanoparticles (Erb et al., 2012). During MASC, a timedependent jamming front moves from the porous mold into the slurry and fixes the position of the platelets in an orientation that is controlled by a rotating magnetic field (Figure 1, step II) (Le Ferrand et al., 2015). In order to produce nacre-like architectures, the rotation plane of the magnetic field was oriented parallel to the mold surface, aligning the platelets horizontally. Scaffolds with oriented platelets were dried and the organic binder was burned out prior to sintering in a hot press (Figure 1, step III). The next step was to re-hydroxylate the ceramic surfaces with a piranha treatment and to graft a methacrylate silane onto the scaffold surface. This coupling agent is expected to later improve the bonding between the scaffold and the polymeric phase (Figure 1, step IV) (Munch et al., 2008).

\subsection{Polymer infiltration}

Composites with three different matrix polymers - poly(lauryl methacrylate) (PLMA), poly(methyl methacrylate) (PMMA) and polyether urethane diacrylate-copoly(2-hydroxyethyl methacrylate) (PUA-PHEMA) - were created by infiltrating porous scaffolds with the respective monomers and polymerizing them in situ (Figure 1, step V) (Launey et al., 2009; Le Ferrand et al., 2015; Munch et al., 2008). The polymer architectures as well as the chemical structure of the respective monomers are sketched in Figure 2. 


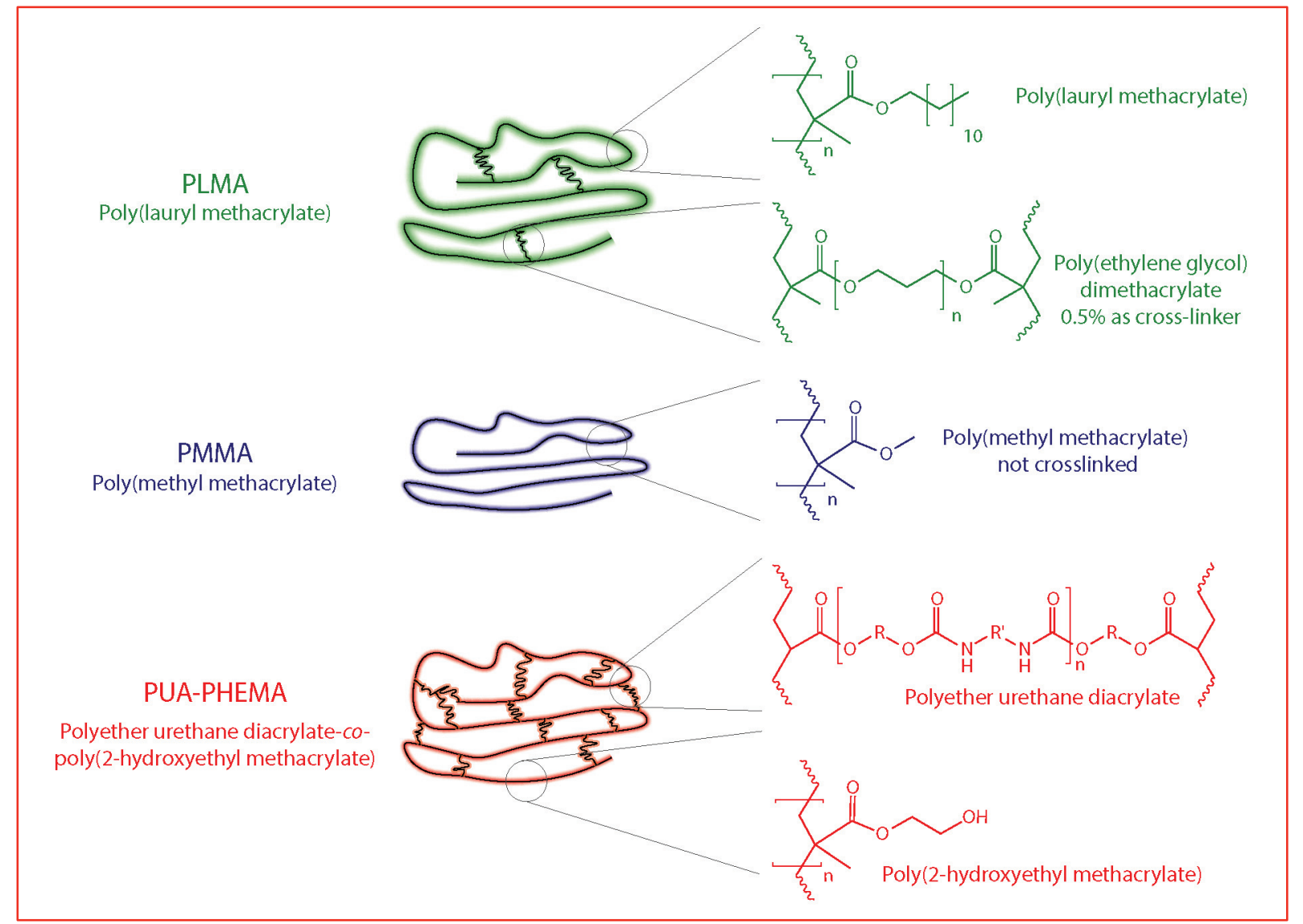

Figure 2: Schematics of polymer structures and their molecular constituents. $R$ and $R^{\prime}$ in PUA-PHEMA form an aliphatic polyether backbone.

For PMMA and PLMA, the polymerization process was conducted in two steps following a procedure described in the literature for PMMA-ceramic composites (Launey et al., 2009; Le Ferrand et al., 2015; Munch et al., 2008). Initially, the reaction conditions were adjusted to increase the probability of PMMA chains being grafted onto the scaffold surface (Launey et al., 2009; Munch et al., 2008). Such chains can then entangle effectively with the polymer that is synthesized in the subsequent bulk polymerization step. The experimental details are given in the supplementary information.

Since PUA-PHEMA is a crosslinked random copolymer, growing linear chains from the scaffold surface was not feasible in this case. Thus, the monomers used to obtain PUA-PHEMA were infiltrated and cured in one step. A homogeneous mixture of the monomers consisting of two parts by weight of polyether urethane diacrylate BR571 
and three parts by weight of 2-hydroxyethyl methacrylate (HEMA) was obtained by strong stirring for 24 h. 1 wt\% V-65 initiator was dissolved in this mixture. $\gamma$-MPS grafted scaffolds were immersed in the monomer-initiator mixture and vacuum of 7 mbar was applied for several hours until no bubbles developed anymore. Subsequently the samples were cured in a $\mathrm{N}_{2}$ filled oven at $50{ }^{\circ} \mathrm{C}$ for $90 \mathrm{~min}$ and post-cured at $80^{\circ} \mathrm{C}$ for $120 \mathrm{~min}$.

To distinguish them from the pure polymers, the composites are named C-PLMA, C-PMMA and C-PUA-PHEMA where necessary. The degree of infiltration (DoI) was determined by a combined thermogravimetric and Archimedes density approach. Bending tests and fracture mechanics tests as well as electron microscopy were performed as described by Le Ferrand et al. (Le Ferrand et al., 2015). Details are also provided in the supplementary information.

\section{Finite Element (FE) simulations}

\subsection{Image treatment}

The cross-section of a PMMA composite was polished by broad-ion-beam milling (IM4000 Hitachi, Japan) and scanning electron micrographs were taken at 10'000x magnification.

The freely available software Fiji was used for all image treatment (Schindelin et al., 2012). An array of 16 SEM images was stitched (Preibisch et al., 2009) together in order to simulate an area large enough to encompass several platelets, while simultaneously maintaining the resolution sufficiently high to enable differentiation between platelets and interconnections (Figure 3a). A bandpass filter, from 4 pixels to 40 pixels, was applied to remove the average gray level difference between the 
individual images. Then, the plugin "trainable segmentation" was used to differentiate the mineral and organic phases. In the final image a median filter of 1 pixel was applied to remove small segmentation artifacts.

\subsection{Numerical model}

The FE model was created using the commercially available software COMSOL. The geometry obtained by image analysis was set to a 2D plane strain model, with two symmetric boundary conditions, a free side and a prescribed displacement on one of the sides (Figure 3c). Due to convergence problems no periodic boundary conditions could be used. A time dependent boundary displacement with a rate of $1 \mu \mathrm{m} . \mathrm{s}^{-1}$ was simulated. Material properties for the inorganic and the organic phase were assigned to the segmented areas obtained from image analysis. Due to the attribution of material parameters based on a binary image, the same constitutive model had to be used for the two parts of the composite. An elasto-plastic law with isotropic hardening represented the best compromise in terms of possibilities, low number of fitted parameters and complexity. 


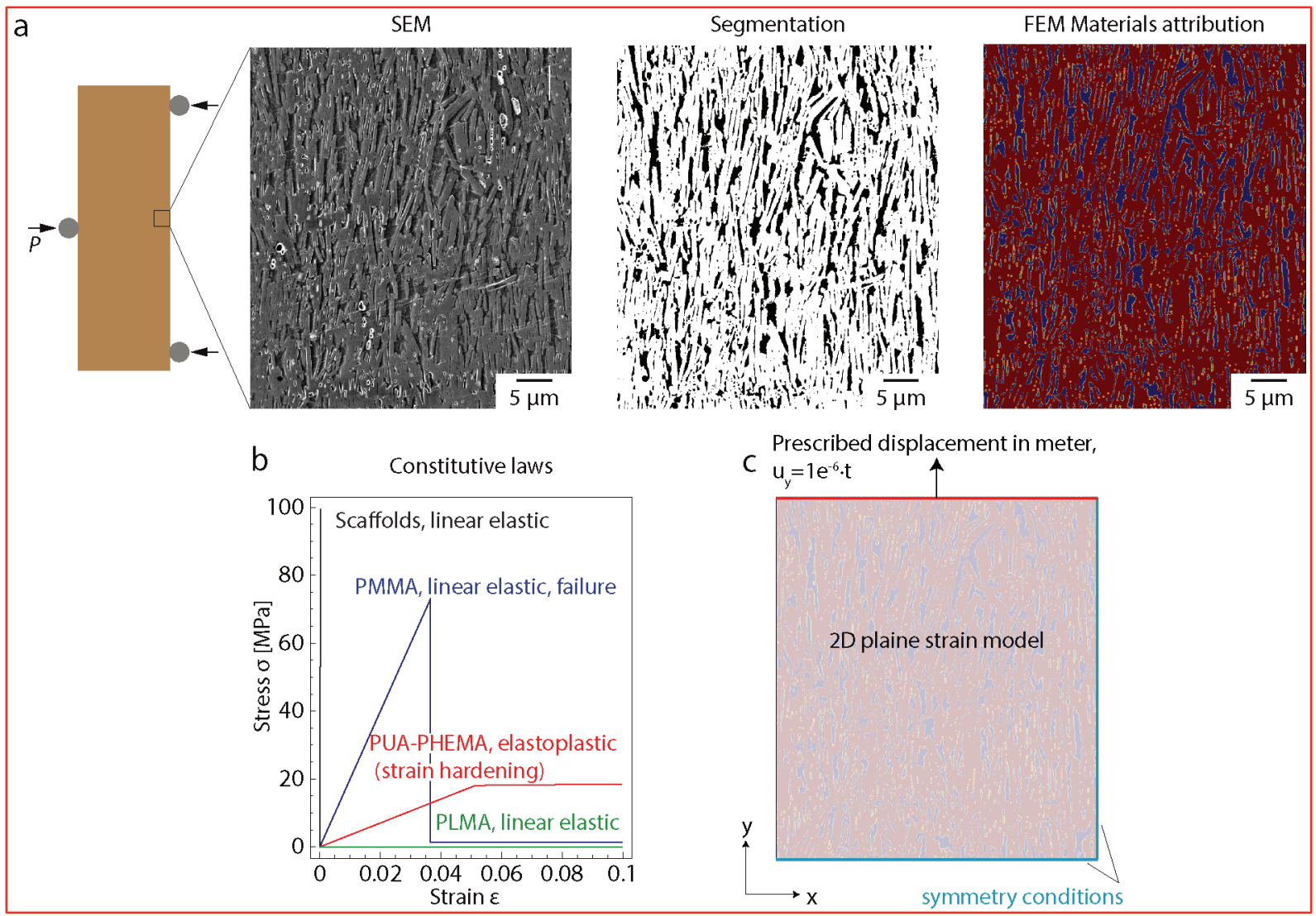

Figure 3: (a) Construction of the FE-model from the actual composite microstructure. Image analysis was used to segment a representative SEM image in two phases: the polymer (blue) and the mineral (red) parts. This image is then used to attribute the material's properties in the model. The loading is simple tension. (b) Constitutive laws used in the model for the different phases. PMMA damage is modeled using a decreasing yield stress after the maximum. (c) Geometry of the 2D plane strain model. The variable $t$ is the elapsed time.

The constitutive model for the inorganic part was purely linear elastic, with a modulus of $400 \mathrm{GPa}$ and a density of $3^{\prime} 970 \mathrm{~kg} \cdot \mathrm{m}^{-3}$, corresponding to pure alumina (Callister and Rethwisch, 2003). The air in the unfilled scaffold was represented arbitrarily by a linear elastic material with a modulus of $0.01 \mathrm{MPa}$ and a density of 1 kg.m-3 ${ }^{-3}$ For numerical reasons, these values could not be equal to zero. The moduli of PMMA and PUA-PHEMA were taken directly from tensile tests, whereas for PLMA the tensile behavior was linearized over the entire deformation range (compare Figure 5 and Table 1). The densities of the polymers were determined using the Archimedes method. For PLMA, a linear elastic model was used, with a modulus of $0.047 \mathrm{MPa}$ and a 
density of $928 \mathrm{~kg} \cdot \mathrm{m}^{-3}$. The stress-strain curve describing the mechanical response of PMMA was split in three sections: a linear elastic part with a modulus of 2.0 GPa until the yield stress of $73 \mathrm{MPa}$, followed by an abrupt decrease in stress to $1 \mathrm{MPa}$ to represent fracture of the polymer. In the last part the stress was kept constant at $1 \mathrm{MPa}$ to avoid convergence issues. The density of PMMA was determined to be $1^{\prime} 150 \mathrm{~kg} \cdot \mathrm{m}^{-3}$. For the PUA-PHEMA polymer, an elastoplastic model was used. The elastic part presents a Young's modulus of $352 \mathrm{MPa}$ until a yield stress of $18 \mathrm{MPa}$ is reached. After this point, an isotropic hardening plasticity model was used with a modulus of $8.7 \mathrm{MPa}$. A density of $1^{\prime} 170 \mathrm{~kg} \cdot \mathrm{m}^{-3}$ was assumed for this polymer. Although more elaborate fracture models have been proposed in the literature (Moës et al., 1999), our simple approach using a realistic microstructure from an SEM image is effective in capturing the essential micromechanical features of the investigated composites.

During the simulations, a first mesh of 50'000 triangular elements was automatically generated by the software. Then, the software refined the mesh taking into account the largest error obtained for each element during a small deformation step. Six consecutive refinement steps were performed until the global error reduction stopped with increasing the number of elements. This final mesh consisted of 1'700'000 elements adapted to the geometry and was used for all subsequent calculations. An implicit time dependent solver (backward differentiation formula, BDF) was used for all the calculations. A stress distribution histogram was computed with Matlab by binning the von Mises stresses of all nodes of the elements in the model.

We choose von Mises stresses to represent the state of our composites since we use them also as yield criterion in the constitutive laws. Alternatively, other stresses could have been used without changing the main conclusions, for instance the first principal stress (see also Figure S4). 


\section{Results}

\subsection{Porous scaffold}

MASC allows for the preparation of bulk ceramic scaffolds with reproducible porosity and a microstructure of aligned alumina platelets that exhibit connecting mineral bridges and nanometer-sized asperities (Figure 4). The samples prepared in this study had a diameter of $20 \mathrm{~mm}$, a thickness of 3 to $4 \mathrm{~mm}$ and were macroscopically homogeneous (Figure 4a). In the electron micrograph of a fracture surface of an unfilled scaffold (Figure 4b), the nacre-like microstructure is easily visible. Platelets in such a bioinspired architecture are horizontally aligned throughout the entire bulk specimen. The electron micrograph in Figure 4c shows a higher magnification of the structure, in which the silica nanoparticles are clearly visible on the alumina platelets. They form nanoasperities that are expected to provide additional interlocking between the polymer phase and the platelets (Le Ferrand et al., 2015; Libanori et al., 2016). When platelets are sufficiently close together, the silica spheres also form sintered mineral bridges and thus create a strong inorganic bond. The relative mineral content of the scaffold was adjusted to $57 \mathrm{vol} \%$, which allows for the formation of a strong percolating ceramic network while still providing enough porosity for the infiltration with monomers leading to the different polymers investigated in this work.

For C-PLMA and C-PMMA specimens a similar DoI of $85 \pm 5 \%$ and $85 \pm 3 \%$ were determined. The nominal $\mathrm{DoI}$ of $102 \pm 4 \%$ indicates complete infiltration for C-PUAPHEMA. Possible reasons for these variations in DoI include differences during the actual infiltration procedure as well as different polymerization shrinkage of the infiltrated monomers. 

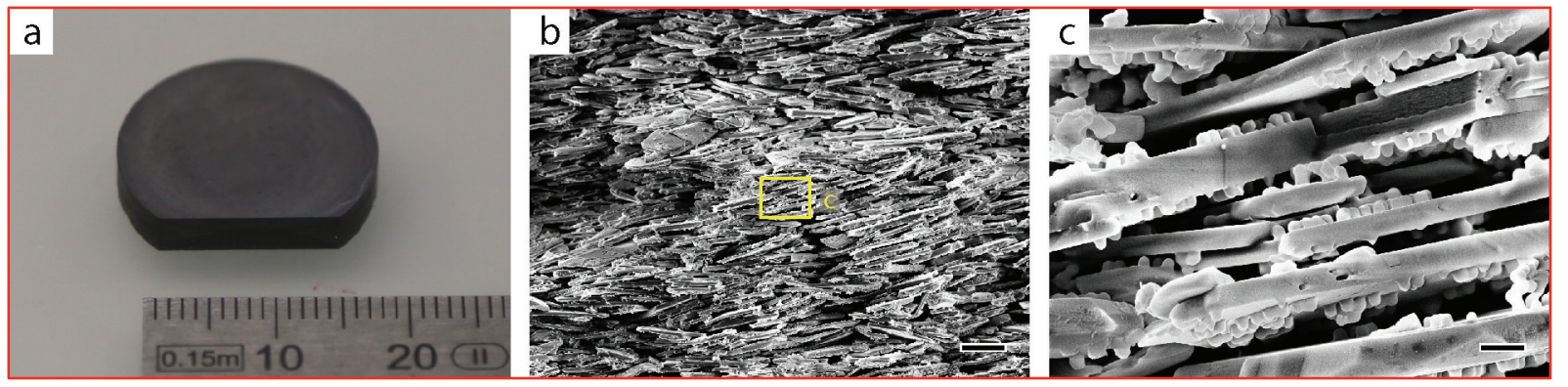

Figure 4: (a) Photograph of a bulk ceramic scaffold after hot pressing. One edge is cut to show the macroscopically homogeneous structure (ruler scale in $\mathrm{mm}$ ) (b,c) SEM images of the fracture surface showing the microstructure of the ceramic scaffolds. Scale bars: (b) $5 \mu \mathrm{m}$, (c) $0.5 \mu \mathrm{m}$.

\subsection{Polymer properties}

The mechanical properties of the three (meth)acrylic polymers that are used to form the organic phase within the ceramic scaffolds span over several orders of magnitude as illustrated in Figure 5 and summarized in Table 1.

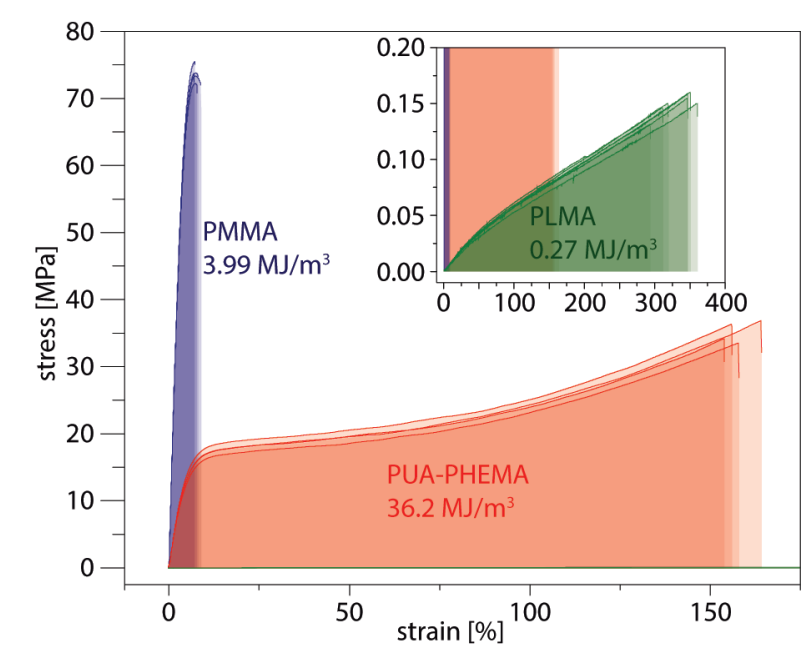

Figure 5: Tensile stress-strain curves of the polymers used as organic phase. The modulus of toughness is noted as the area under the curve.

Table 1: Tensile properties of the polymers used as organic phase.

\begin{tabular}{ccccc} 
Polymer & $\begin{array}{c}\text { Elastic modulus } \\
{[\mathrm{MPa}]}\end{array}$ & $\begin{array}{c}\text { Tensile strength } \\
{[\mathrm{MPa}]}\end{array}$ & $\begin{array}{c}\text { Strain at failure } \\
{[\%]}\end{array}$ & $\begin{array}{c}\text { Modulus of } \\
\text { toughness } \\
{\left[\mathrm{MJ} / \mathrm{m}^{3}\right]}\end{array}$ \\
\hline PLMA & $0.077 \pm 0.05$ & $0.15 \pm 0.01$ & $333.0 \pm 24.7$ & $0.27 \pm 0.03$ \\
PMMA & $2008 \pm 25$ & $73.7 \pm 1.2$ & $7.7 \pm 0.8$ & $3.99 \pm 0.53$ \\
PUA-PHEMA & $352 \pm 2$ & $35.2 \pm 1.6$ & $157.9 \pm 4.4$ & $36.21 \pm 1.9$
\end{tabular}


Pure PLMA is a highly viscous and sticky liquid at room temperature and is thus crosslinked with $0.5 \mathrm{wt} \%$ poly(ethylene glycol)dimethacrylate (PEGDMA) to result in a soft and weak but stretchable elastomer exhibiting an elastic modulus of $0.077 \mathrm{MPa}$ and a tensile strength of $0.15 \mathrm{MPa}$. The strain at failure is the highest of all three investigated polymers with a value of $333 \%$. The modulus of toughness calculated from the area under the stress-strain curve is low and reaches only $0.27 \mathrm{MJ} / \mathrm{m}^{3}$.

PMMA is a well-established glassy polymer that reaches a high strength of 73 $\mathrm{MPa}$ and a high stiffness of $2 \mathrm{GPa}$. Despite its low strain at failure of $7.7 \%$, the modulus of toughness is $4 \mathrm{MJ} / \mathrm{m}^{3}$, which is one order of magnitude higher than the one of PLMA.

In polyurethane acrylates, the wide range of mechanical properties covered by polyurethanes is combined with the chemical versatility of acrylate polymers. We chose the combination of a polyether urethane diacrylate and HEMA as reactive diluent. The resulting polymer, PUA-PHEMA, shows non-linear elastic behavior with an elastic modulus of $352 \mathrm{MPa}$, a tensile strength of $35 \mathrm{MPa}$ and a strain at failure of $158 \%$. These values lie at an intermediate level between those of PLMA and PMMA. In combination however the properties of PUA-PHEMA lead to a modulus of toughness of $36.2 \mathrm{MJ} / \mathrm{m}^{3}$, which is one and two orders of magnitude higher than the values measured for PMMA and PLMA, respectively.

Based on the polymer strength data reported above as well as the aspect ratio and previously estimated strength of the alumina platelets, a simplified shear lag analysis would suggest that all the composites investigated here should fail under platelet pull-out mode (Bonderer et al., 2008). Such a simplified analysis should be taken with care, however, since it does not consider the high volume fraction of platelets of our composites nor the presence of mineral bridges. This should only be valid if the 
fracture process occurs through the rupture of the mineral bridges first, which would later lead to the microstructure assumed in the shear lag model comprising isolated platelets surrounded by a continuous polymer matrix.

\subsection{Mechanical properties of nacre-like composites}

The flexural properties and fracture behavior of composites containing each of the studied polymers were characterized by three-point bending (3 Pt.B.) and single edge notched beam (SENB) tests. An unfilled scaffold was evaluated in the same way to provide a reference. The results of these tests clearly show that the mechanical properties of the composites are remarkably influenced by the choice of the polymer used as organic phase (Figure 6 and Table 2).

Table 2: Mechanical properties of the examined nacre-like composites

\begin{tabular}{|c|c|c|c|c|c|}
\hline Composite & $\begin{array}{c}\text { Flexural modulus } \\
\qquad E_{f} \\
{[\mathrm{GPa}]}\end{array}$ & $\begin{array}{c}\text { Fracture strength } \\
\sigma_{f} \\
{[\mathrm{MPa}]}\end{array}$ & $\begin{array}{c}\text { Strain at failure } \\
\varepsilon \\
{[\%]}\end{array}$ & $\begin{array}{c}\text { Fracture toughness } \\
\qquad K_{/ C} \\
{\left[\mathrm{MPa} \cdot \mathrm{m}^{1 / 2}\right]}\end{array}$ & $\begin{array}{c}\text { Work of fracture } \\
\qquad \gamma_{\text {wof }} \\
{\left[\mathrm{J} / \mathrm{m}^{2}\right]}\end{array}$ \\
\hline Scaffold & $35.0 \pm 8.1$ & $99.7 \pm 9.9$ & $0.27 \pm 0.06$ & $1.88 \pm 0.21$ & $42.6 \pm 8.7$ \\
\hline C-PLMA & $35.3 \pm 5.4$ & $99.9 \pm 10.3$ & $0.25 \pm 0.02$ & $1.43 \pm 0.30$ & $42.4 \pm 7.8$ \\
\hline C-PMMA & $36.6 \pm 6.7$ & $182.3 \pm 13.8$ & $0.43 \pm 0.06$ & $2.40 \pm 0.35$ & $110.0 \pm 4.2$ \\
\hline C-PUA-PHEMA & $36.8 \pm 3.8$ & $168.1 \pm 17.5$ & $0.44 \pm 0.03$ & $3.39 \pm 0.28$ & $156.2 \pm 1.7$ \\
\hline
\end{tabular}

Interestingly, representative flexural stress-strain curves displayed in Figure 6a indicate that the ceramic scaffold dominates the composite stiffness, since the three composites and the unfilled scaffold exhibit an almost identical flexural modulus of around $35 \mathrm{GPa}$. In terms of strength and strain at failure, the samples can be classified in two groups: (1) the unfilled scaffold and the PLMA composite and (2) the PMMA and PUA-PHEMA composites. The unfilled scaffold and the PLMA composite reach almost identical flexural strength and strain at failure values of $100 \mathrm{MPa}$ and around $0.27 \%$, respectively. This indicates that the soft and weak PLMA does not influence the flexural 
performance of this type of composites. In contrast, both PMMA and PUA-PHEMA infiltrated composites achieve a higher strain at failure of around $0.43 \%$ and an increased flexural strength of $182 \mathrm{MPa}$ and $168 \mathrm{MPa}$, respectively.
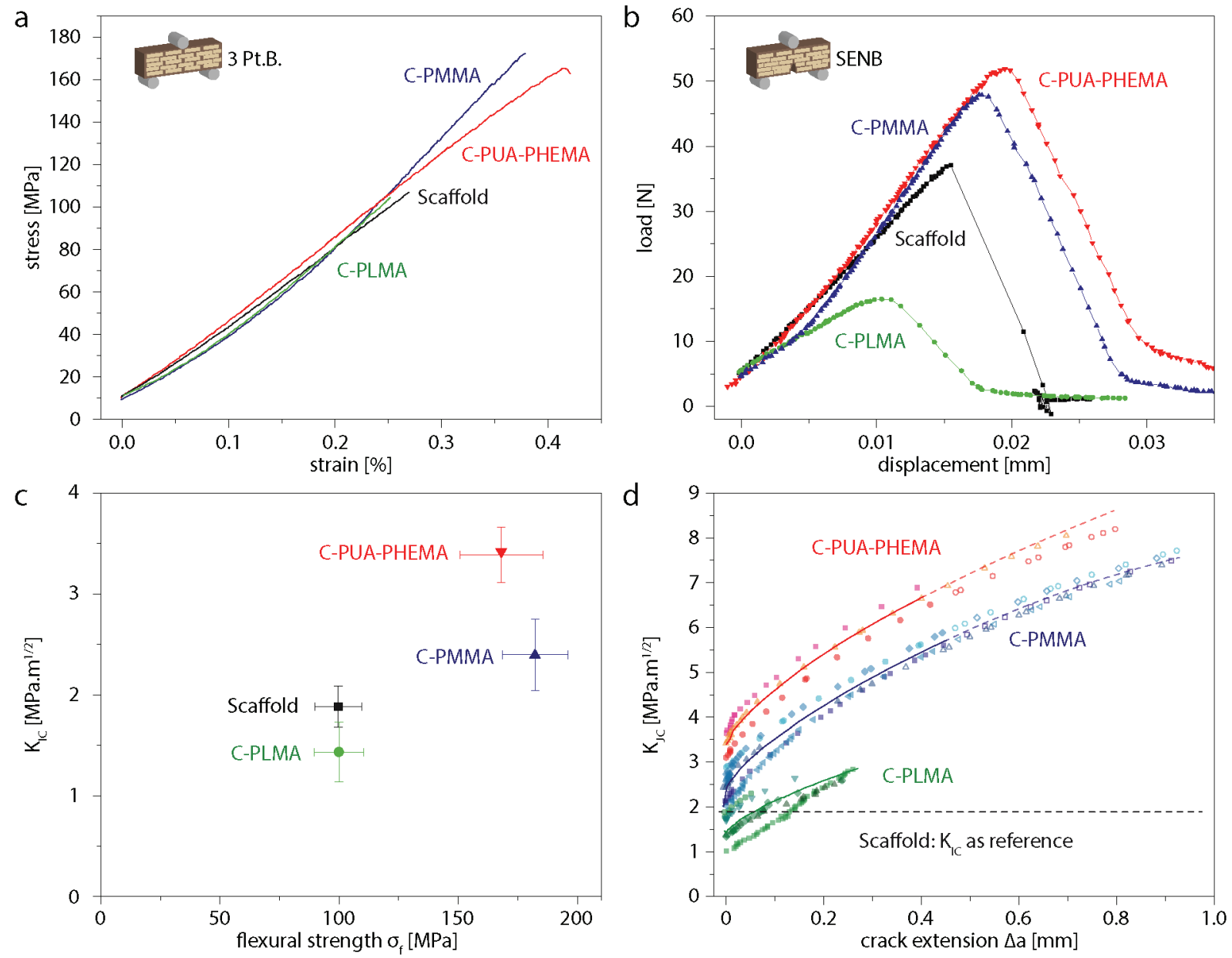

Figure 6: Mechanical characterization of the nacre-like ceramic-polymer composites with different polymer matrices. (a) Representative stress-strain curves from 3-point-bending (3 Pt.B.) tests. (b) Representative load-displacement curves from single edge notched beam (SENB) tests. Note that the measured specimens differ slightly in dimensions. (c) Plot of fracture toughness $K_{I C}$ as function of the flexural strength $\sigma_{f}$. Data points represent the average for three or more specimens while error bars indicate the standard deviation. (d) Crack growth resistance curves (R-curves) for the different composites determined from the SENB curves. The $K_{I C}$ value for the unfilled scaffold is given as reference. Solid lines were obtained by averaging over the data points shown. Filled symbols display data points that lie within the ASTM-limits. Broken lines and empty symbols indicate ASTM-invalid values shown on the basis of J-integral considerations (see SI for details) (Launey et al., 2010; Launey et al., 2009; Naglieri et al., 2015). 
Representative load-displacement curves from SENB fracture toughness measurements are shown in Figure 6b. Note that the absolute values in these curves should not be compared quantitatively since the specimens used for the measurements display slightly different dimensions. Instead, these plots are shown to qualitatively describe the overall fracture behavior of the pure scaffold and the three composites. The scaffold exhibits elastic response and fails catastrophically. Some specimens showed limited non-linear-elastic deformation before brittle failure. This specimen dependent behavior is probably a consequence of the relatively high porosity of $43 \%$. By contrast, crack propagation in the three polymer-infiltrated composites is stable after the maximum strength is reached, as indicated by the continuous decay in strength after the initial elastic deformation.

The SENB results were used to evaluate the fracture mechanics of the composites quantitatively. The fracture toughness at crack initiation, $K_{I C}$, is plotted against the flexural strength in Figure 6c. In addition to identical strength values, the unfilled scaffold and the PLMA composite also show a similar fracture toughness. Interestingly, the $K_{I C}$ value of the PLMA composite (1.4 MPa.m ${ }^{1 / 2}$ ) is even slightly lower than the one of the scaffold (1.9 MPa.m ${ }^{1 / 2}$ ), despite the stable crack propagation in the composite. The scaffold of the composite and its mineral bridges were possibly weakened during the piranha treatment before the silanization process, leading to the lowered initial toughness. In any case, the similar toughness of the scaffold and C-PLMA is further supported by the almost identical work of fracture $\gamma_{\text {wof }}$ of the two materials (compare Table 2). Besides a higher strength, the composites infiltrated with PMMA and PUAPHEMA also exhibit higher fracture toughness values of 2.4 and $3.4 \mathrm{MPa} \cdot \mathrm{m}^{1 / 2}$, respectively. This trend is reflected also in the increased $\gamma_{\text {wof }}$ values of $110 \mathrm{~J} / \mathrm{m}^{2}$ for CPMMA and $156 \mathrm{~J} / \mathrm{m}^{2}$ for C-PUA-PHEMA. 
In agreement with the qualitative analysis of the SENB load-displacement curves, all three types of composites show rising crack growth resistance curves (R-curves) (Figure 6d). This is quite remarkable since the ceramic phase that dominates the elastic modulus of the composite is mostly brittle and only limited, embryonic R-curves can be constructed from the SENB data of the unfilled scaffold (compare supplementary information). Such results indicate that the presence of a polymer phase activates toughening mechanisms that increase the energy dissipated within the material during crack propagation. The first part of the displayed R-curves - drawn with a solid line and filled symbols - shows data obtained within the ASTM valid range of crack extension ( $\Delta a_{\max }=0.25 b, b$ being the initial material ligament) (ASTM, 2015). The values indicated with dashed lines and open symbols in the second part of the curve lie outside the conservative limits of the ASTM standard. The ASTM limit ensures that the measured toughness represents a material property and thus should be independent of specimen geometry and size. This limit is particularly critical in materials exhibiting large-scale bridging in the wake of the propagating crack, which is often associated with a discontinuity in the $J(\Delta a)$-curve (see SI and references for details) (Launey et al., 2010; Launey et al., 2009; Naglieri et al., 2015). As the J-integrals we measure do not show such a discontinuity, the toughness values that lie beyond the ASTM limit are physically meaningful but to remain conservative should be treated with caution.

\subsection{Fracture surfaces and toughening mechanisms}

To gain further insight into the microstructure and the toughening mechanisms responsible for the rising crack growth resistance shown by the polymer-infiltrated scaffolds, we examined the fracture surface of tested specimens by electron microscopy (Figure 7). 
For the PLMA composites (Figure 7a), we observe that the polymer entirely covers the scaffold even after fracture. As there is no visible sign of interfacial damage, the crack likely propagates through the polymer phase. Such propagation leads to extensive polymer deformation and stretching, as pointed out by the green arrows in Figure 7a. This indicates that the soft and sticky PLMA acts as a viscoelastic glue that is able to maintain a minimum integrity in the microstructure of the scaffold despite lacking load-bearing capacity.
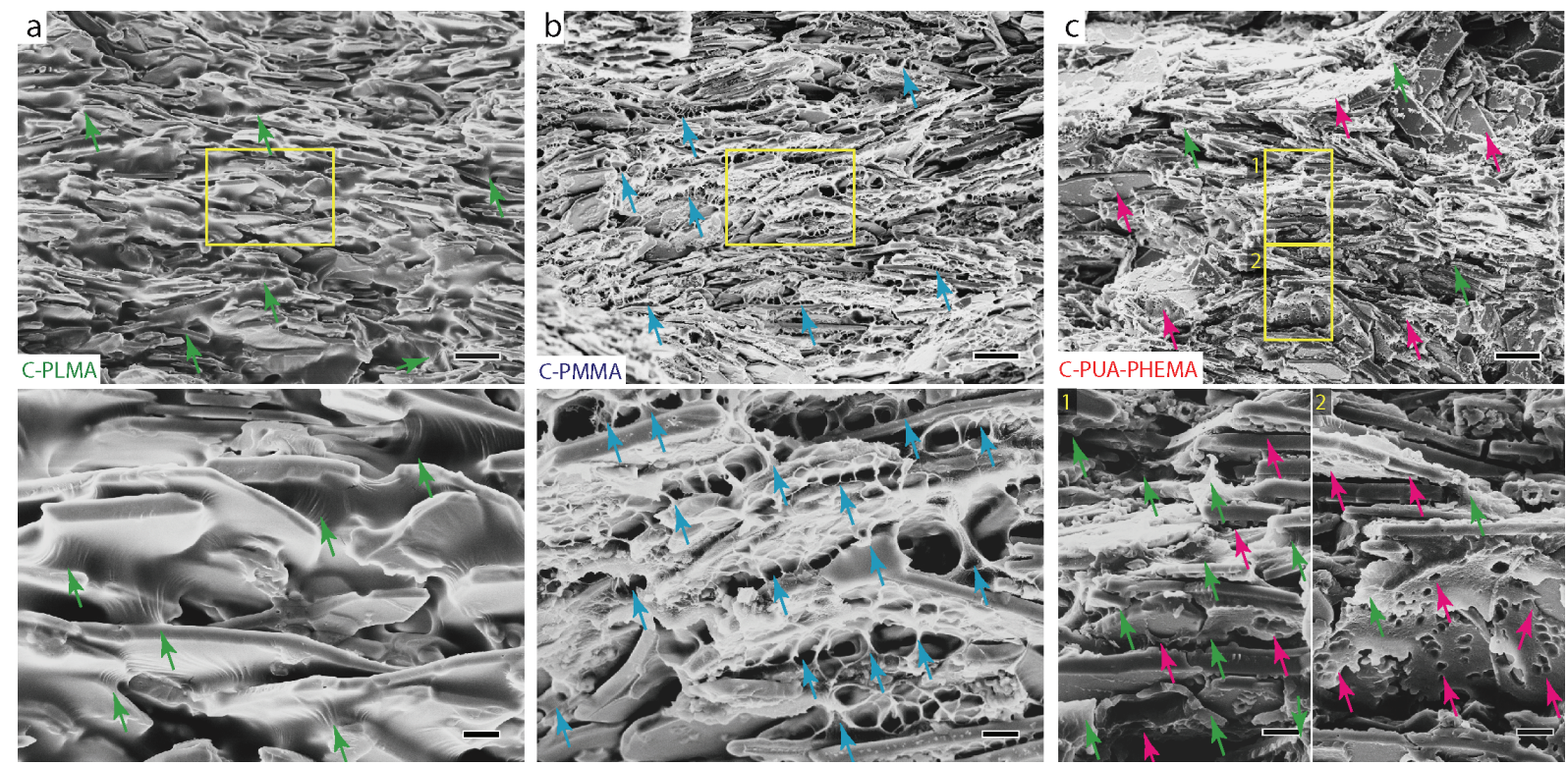

Figure 7: SEM images of fracture surfaces of nacre-like composites with different polymers: (A) C-PLMA, (B) C-

PMMA and (C) C-PUA-PHEMA. Scale bars: $5 \mu \mathrm{m}$ (top row) and $1 \mu \mathrm{m}$ (bottom row). Green arrows indicate extensive polymer deformation or ligament formation; blue arrows indicate polymer fibrillation and microvoid formation; pink arrows point to signs of delamination or polymer-ceramic interlocking.

The fracture surface of a PMMA composite (Figure 7b) shows that also in this case the scaffold is covered with polymer and thus the crack propagates mostly through the organic phase. More importantly, the abundance of polymer fibrils formed between the ceramic platelets reveals microvoid formation as an important failing mechanism of the PMMA phase (highlighted by blue arrows in Figure 7b). 
As opposed to the PLMA and PMMA-containing specimens, the fracture surface of the PUA-PHEMA composite (Figure 7c) suggests that crack propagation in this case does not occur within the organic phase but predominantly at the interface between the polymer and the ceramic scaffold. This is visible in the areas indicated by pink arrows in Figure $7 c$, where either polymer delamination or imprints of the silica nanoasperities are observed. Despite delamination, the polymer was visibly deformed during the fracture process. One possible reason for the delamination might be the one-step polymerization carried out for the PUA-PHEMA composite, which could lead to a weaker interfacial bonding compared to the two-step polymerization used in the other cases. Another tentative explanation for the observed delamination is the intrinsic mechanical behavior of PUA-PHEMA. The high strength combined with high deformability of this polymer might have prevented it from breaking before failure of the interface. During delamination, the relative displacement of the two new surfaces is expected to dissipate additional energy by friction between the nanoasperities and the polymer.

Interestingly, no evidence of platelet fracture was found in any of the observed fracture surfaces, suggesting that the mineral bridges are likely to be the first and only inorganic structural features to rupture during the fracture process. This allows for the onset of toughening mechanisms like platelet pull-out, crack deflection, interfacial delamination and plastic deformation of the organic phase.

\section{Finite Element analysis}

Computer simulations were performed to complement our analysis of the mechanics of the investigated nacre-like composites and to help us understand aspects that cannot be assessed by experimental techniques. We used a finite element (FE) model to evaluate the stress distributions that occur within the organic and inorganic 
phases during mechanical loading of the composites and of the unfilled scaffold (Figure 8).

Von Mises stress distribution histograms are plotted in Figure 8a for a global strain of $0.27 \%$. This is the strain at failure of the unfilled scaffold and is thus used as a reference. The stress distributions can be divided into four sections: (i) below $100 \mathrm{MPa}$, the stresses are carried mainly by the polymer phase up to its respective yield stress; (ii) between 100 and $1000 \mathrm{MPa}$, the stresses are found in the platelets; (iii) just above 1000 $\mathrm{MPa}$ and (iv) around $4000 \mathrm{MPa}$, the stresses are localized in the platelet connections and mineral bridges (see supplementary information for details on the assignment of these sections).

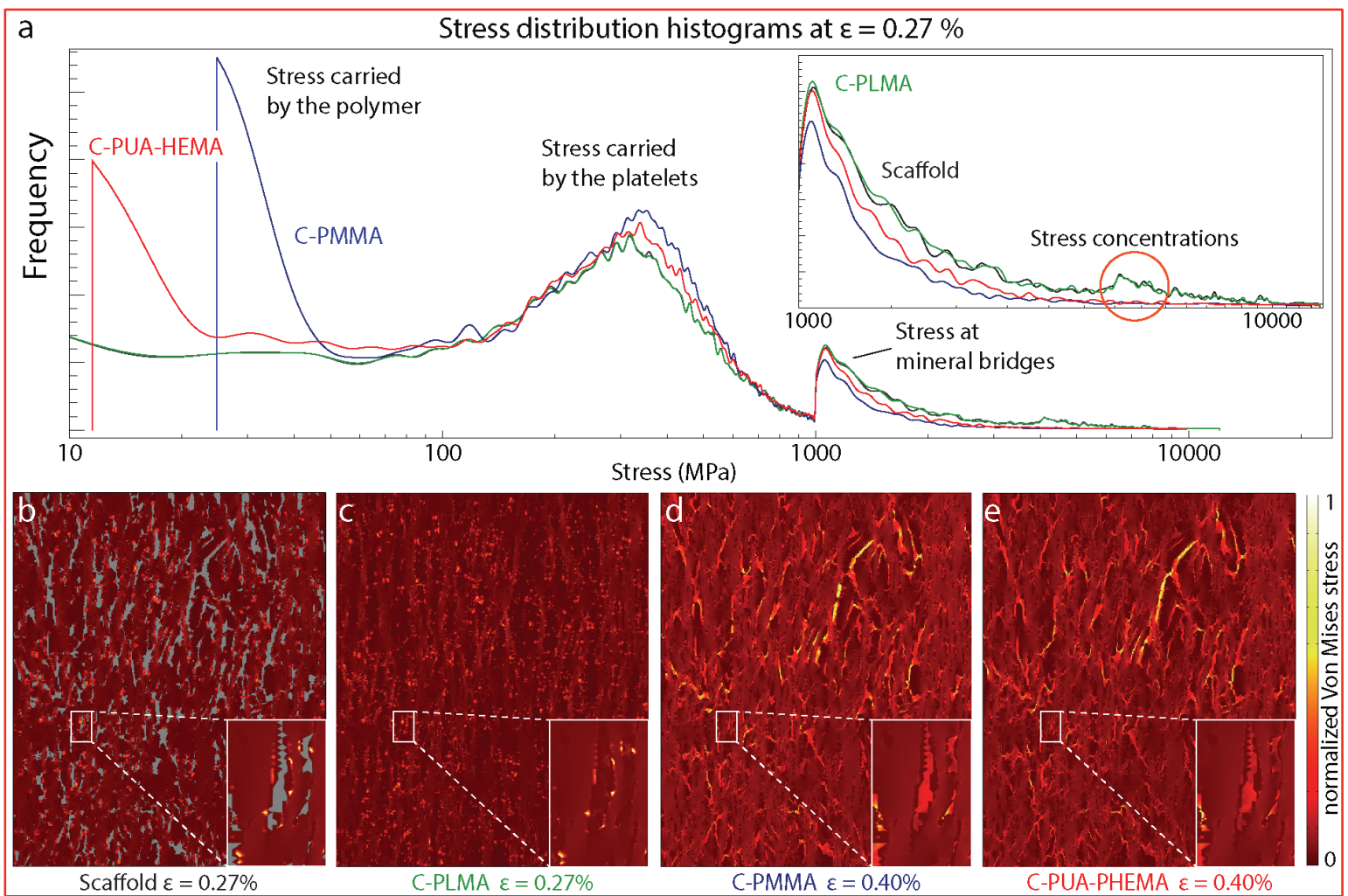

Figure 8: (a) Simulated von Mises stress distribution in the composites at $0.27 \%$ strain. (b-e) Normalized von Mises stresses at the respective maximum experimental strain of each particular composite. The stress in the ceramic scaffolds is divided by the maximum stress simulated for the pure scaffold whereas the stress in the polymer is divided by its maximum tensile strength. 
In agreement with the trends observed for the fracture toughness and strength, the stress distribution histograms simulated for the unfilled scaffold and the PLMA composite are almost identical. In both cases the stresses are carried almost exclusively by the ceramic phase and stress concentrations can be found in the platelet connections (Figure 8a, inset). By contrast, the organic phase of composites containing PMMA and PUA-PHEMA carries stresses up to their maximum strengths. Due to the higher Young's modulus of PMMA, the composite with this polymer shows a higher stress peak than the one with PUA-PHEMA. Remarkably, no stress concentrations can be found in the scaffolds of both composites.

Normalized von Mises stress maps for all composites at their respective strain at failure are displayed in Figure 8b-e. Normalization was carried out as follows: the stress in the mineral phase is divided by the maximum stress found in the unfilled scaffolds, whereas the stresses developed in the organic phases are normalized by the tensile strength of the respective polymer. Bright spots observed in the simulated stress maps of the unfilled scaffold and the PLMA composite (insets in Figure 8b,c) indicate the sites of stress concentrations illustrated in Figure 8a (inset). Conversely, the PMMA and PUAPHEMA composites do not display such local stress concentration spots in the mineral phase (insets in Figure 8d,e). In these two cases, the distribution of stresses between the organic and inorganic phases looks almost identical, despite the different mechanical response of the two polymers (linear elastic and elastoplastic). Because of the linear elastic and brittle nature of the organic phase (Figure 3b), the PMMA-containing composite was the only one in which the strain at failure of the polymer was locally surpassed (see supporting information for strain map). This is in agreement with the microvoid formation experimentally observed within the organic phase of such composites (Figure 7b). 


\section{Discussion}

The 3-point bending results (Figure 6a) suggest that the ceramic scaffold interconnected through mineral bridges dominates the stiffness of the examined nacrelike composites. The strength and the strain at failure of the composites however can be improved by infiltrating a load-bearing polymer into the porous ceramic structure. For the unfilled scaffold and the PLMA composite, the negligible stiffness of the secondary phases (air and PLMA) induces the appearance of local stress concentrations at the platelet connections and mineral bridges. By contrast, the higher stiffness of PMMA and PUA-PHEMA enables a more effective and homogeneous stress distribution, consequently avoiding such stress concentrations (Figure 8a). As a result, the composites with these polymers can sustain a higher overall mechanical load before failure.

The crack initiation toughness $\left(K_{I C}\right)$ of the composites is also strongly influenced by the polymer phase (Figure 6c). To identify correlations between the composite toughness and the mechanical properties of the polymer, we plot in Figure 9a-c the $K_{I C}$ values of the composites as a function of the elastic modulus, strain at failure and modulus of toughness of the polymer matrix. The results suggest that the composite $K_{I C}$ correlates strongly with the modulus of toughness of the different polymers (Figure 9c). We therefore hypothesize that plastic deformation of the polymer occurs at the crack tip even under fast fracture conditions, similarly to the localized plasticity present in metals (Miserez et al., 2004). For PLMA, the plastic contribution to the composite $K_{I C}$ is negligible as a consequence of its low modulus of toughness (Figure 6c). In contrast, PMMA undergoes significant deformation at high stress levels during fracture, as indicated by the microvoid formation observed by fractography (Figure $7 \mathrm{~b}$ ) and in the FE model (Figure S6). This dissipates energy in front of the crack tip and increases the 
$K_{I C}$ (Launey et al., 2009; Launey and Ritchie, 2009; Wegst et al., 2015). PUA-PHEMA provides an even more efficient intrinsic toughening arising from a higher deformation at intermediate stress levels possibly combined with interfacial delamination and friction around the crack tip (Figure 7c). The higher DoI measured for this composite also contributes to its outstanding mechanical performance. These features let composites with PUA-PHEMA reach the highest $K_{I C}$ value among the studied compositions.
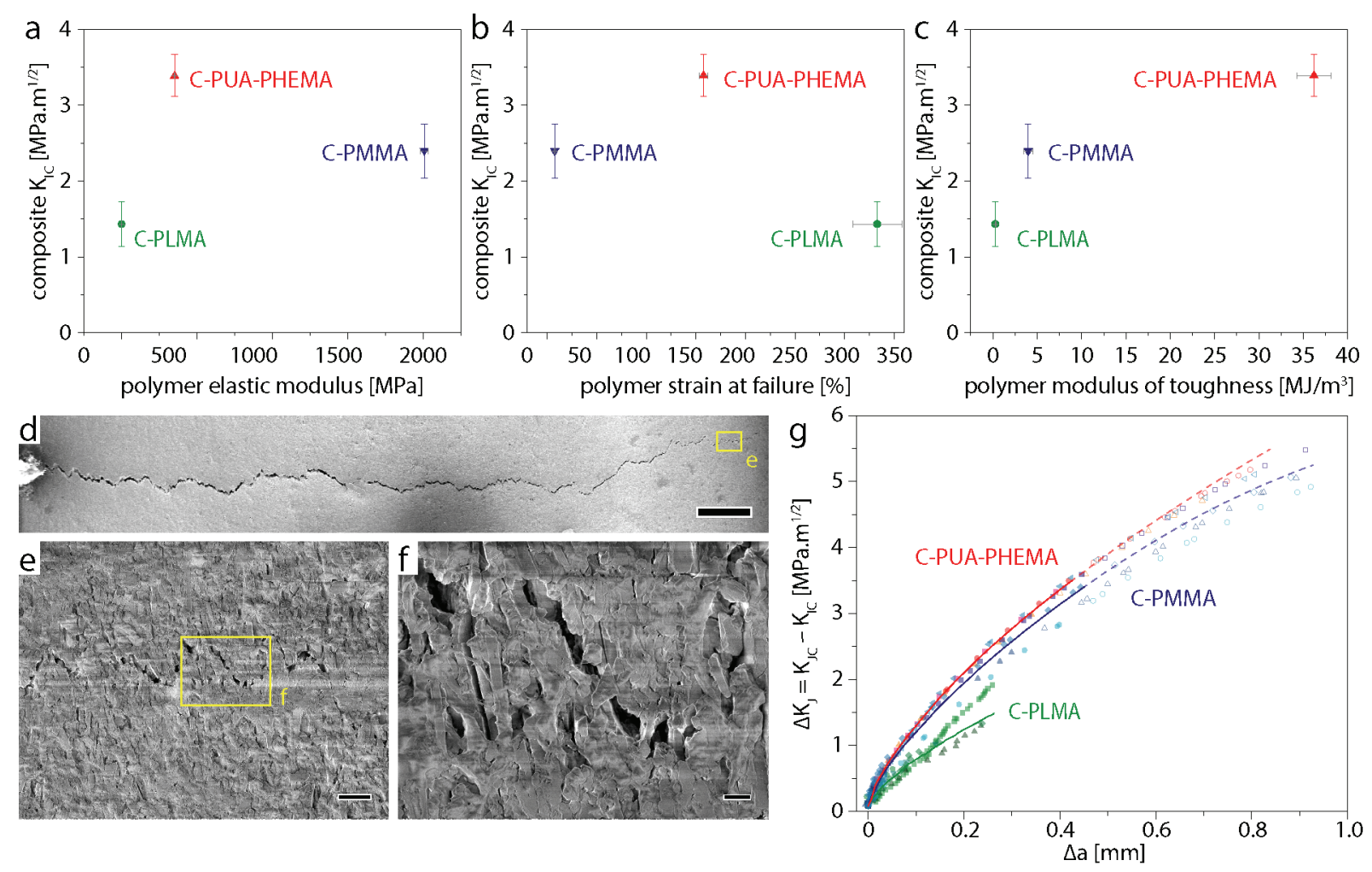

Figure 9: (a-c) Crack initiation toughness, $K_{I C}$, of the composite as function of (a) elastic modulus, (b) strain at failure and (c) modulus of toughness of the polymer matrix. (d-f) Electron micrographs showing a typical crack path in a SENB specimen of the PUA-PHEMA composite at different magnifications. Scale bars: (d) 100 $\mu \mathrm{m}$, (e) $5 \mu \mathrm{m}$ and (f) $1 \mu \mathrm{m}$. (d) Relative toughness increase as function of the crack size. Full and empty symbols indicate ASTM-valid and -invalid data points; solid and broken lines indicate averages over the data points shown.

After crack initiation, further energy dissipating mechanisms are necessary to stabilize the crack growth and to observe a rising R-curve. The fact that hardly any stable crack growth is possible in the porous scaffold indicates that mineral bridges and 
nanoasperities alone are not enough to toughen the material at this low volume fraction of inorganic phase (57 vol\%). Interestingly, filling the pores with a weak and soft polymer like PLMA is already sufficient to significantly stabilize crack propagation, as indicated by the rising R-curve behavior shown in Figure 6d. This suggests that the incorporation of a compliant polymer phase into the scaffold gives rise to additional toughening mechanisms. Indeed, local crack deflection over a few platelet diameters can be observed in the composites with all polymers. Intact material ligaments and pulledout platelets bridge the crack wake, as shown in Figure 9d-f for a composite with PUAPHEMA as an illustrative example. These toughening mechanisms are well known from artificial and natural composites (Launey and Ritchie, 2009; Meyers et al., 2008a; Wegst et al., 2015). Additionally, the abrupt variation in the local Young's modulus as a crack propagates from the ceramic to the polymer phase has also been suggested as an important mechanism that reduces the driving force for crack propagation in lamellar structures (Kolednik et al., 2011).

To better evaluate the resistance of the composites against crack propagation, we compare our data in terms of the net increase in fracture toughness as a function of crack extension: $\Delta K_{J}(\Delta a)=K_{J C}(\Delta a)-K_{I C}$ (Figure 9g) (Barthelat and Rabiei, 2011). The almost identical shape of the $\Delta K_{J}(\Delta a)$ curves of PMMA and PUA-PHEMA composites suggest that the observed difference in the absolute fracture toughness of these two composites ( $K_{J C}$ in Figure $6 \mathrm{~d}$ ) is primarily caused by the distinct initial crack resistances $K_{I C}$ of the materials, which in turn correlate well with the moduli of toughness of the polymers. Given the very similar net increase in fracture toughness, the energy dissipating mechanisms active in these composites during crack propagation seem to be dominated by the ceramic phase. The infiltration with PLMA is sufficient to activate these toughening mechanisms to a certain extent despite being such a soft and weak 
polymer. However, our results indicate that the presence of a strong and tough organic phase (PMMA or PUA-PHEMA) is key to benefit from the full potential of the mechanisms programmed within the scaffold design. Such strong and tough polymer matrices reduce stress concentrations and promote arresting mechanisms that allow the crack to extensively interact with the inorganic microstructure.

\section{Conclusion}

We experimentally show that the polymer phase plays an important role in the mechanical performance of scaffold-based nacre-like composites with intermediate volume fraction of mineral phase. Stiffer polymers like polyether urethane diacrylate-copoly(2-hydroxyethyl methacrylate) (PUA-PHEMA) and poly(methyl methacrylate) (PMMA) were found to significantly increase the composite strength by generating a more homogenous stress distribution and avoiding the formation of stress concentrations at the mineral bridges and platelet connections. Besides stiffness, the modulus of toughness of the polymer phase also affects strongly the mechanical response of the nacre-like composite. The tougher polymers PUA-PHEMA and PMMA lead to composites with enhanced $K_{I C}$-values as compared to unfilled scaffolds or specimens containing the weaker poly(lauryl methacrylate) (PLMA) phase. By contrast, additional toughening during crack propagation (R-curve) arises primarily from mechanisms embedded within the scaffold architecture. However, our experimental data indicate that a minimum strength and toughness of the polymer is necessary to take full advantage of the toughening mechanisms programmed in the scaffold. These results indicate that a strong and stiff organic phase with a high modulus of toughness should be considered for designing bioinspired nacre-like polymer composites with improved strength and fracture toughness at an intermediate ceramic content. 


\section{Acknowledgements}

We are grateful to Hortense Le Ferrand for the help with the broad-ion-beam polishing and to ScopeM at ETH Zürich for providing the necessary specimen preparation and microscopy facilities. We thank Dr. Ofer Tevet for setting up the ultra sound equipment for elastic modulus measurements. We also acknowledge insightful discussions with Dr. Anna Evans, Madeleine Grossman, Marianne Sommer, Erik Poloni and Dr. Kunal Masania. Finally, T.N., D.K. and F.B. thank the financial support from ETH Zurich (ETHIIRA grant number 0-20676-10), the Air Force Office of Scientific Research (grant FA8655-13-1-3002) and the Swiss National Science Foundation (grant 200020_146509), respectively.

\section{References}

Askarinejad, S., Rahbar, N., 2015. Toughening mechanisms in bioinspired multilayered materials. J. R. Soc. Interface 12, 20140855.

ASTM E1820-15, Standard Test Method for Measurement of Fracture Toughness, ASTM International, West Conshohocken, 2015.

Barthelat, F., 2014. Designing nacre-like materials for simultaneous stiffness, strength and toughness: Optimum materials, composition, microstructure and size. J. Mech. Phys. Solids 73, 22-37.

Barthelat, F., Dastjerdi, A.K., Rabiei, R., 2013. An improved failure criterion for biological and engineered staggered composites. J. R. Soc. Interface 10, 20120849.

Barthelat, F., Rabiei, R., 2011. Toughness amplification in natural composites. J. Mech. Phys. Solids 59, 829-840.

Barthelat, F., Tang, H., Zavattieri, P.D., Li, C.M., Espinosa, H.D., 2007. On the mechanics of mother-of-pearl: A key feature in the material hierarchical structure. J. Mech. Phys. Solids 55, 306-337.

Begley, M.R., Philips, N.R., Compton, B.G., Wilbrink, D.V., Ritchie, R.O., Utz, M., 2012. Micromechanical models to guide the development of synthetic 'brick and mortar' composites. J. Mech. Phys. Solids 60, 1545-1560. 
Behr, S., Vainio, U., Müller, M., Schreyer, A., Schneider, G.A., 2015. Large-scale parallel alignment of platelet-shaped particles through gravitational sedimentation. Sci. Rep. 5, 9984.

Bezares, J., Asaro, R.J., Hawley, M., 2008. Macromolecular structure of the organic framework of nacre in Haliotis rufescens: Implications for growth and mechanical behavior. J. Struct. Biol. 163, 61-75.

Bezares, J., Asaro, R.J., Hawley, M., 2010. Macromolecular structure of the organic framework of nacre in Haliotis rufescens: Implications for mechanical response. J. Struct. Biol. 170, 484-500.

Bonderer, L.J., Studart, A.R., Gauckler, L.J., 2008. Bioinspired Design and Assembly of Platelet Reinforced Polymer Films. Science 319, 1069-1073.

Bouville, F., Maire, E., Meille, S., Van de Moortele, B., Stevenson, A.J., Deville, S., 2014. Strong, tough and stiff bioinspired ceramics from brittle constituents. Nat. Mater. 13, 508-514.

Callister, W.D., Rethwisch, D.G., 2003. Materials Science and Engineering: An Introduction, 6th ed. Wiley, New York.

Dastjerdi, A.K., Rabiei, R., Barthelat, F., 2013. The weak interfaces within tough natural composites: Experiments on three types of nacre. J. Mech. Behav. Biomed. Mater. 19, 5060 .

Deville, S., Saiz, E., Nalla, R.K., Tomsia, A.P., 2006. Freezing as a Path to Build Complex Composites. Science 311, 515-518.

Dwivedi, G., Flynn, K., Resnick, M., Sampath, S., Gouldstone, A., 2015. Bioinspired Hybrid Materials from Spray-Formed Ceramic Templates. Adv. Mater. 27, 3073-3078.

Ekiz, O.O., Dericioglu, A.F., Kakisawa, H., 2009. An efficient hybrid conventional method to fabricate nacre-like bulk nano-laminar composites. Mater. Sci. Eng. C 29, 2050-2054.

Erb, R.M., Libanori, R., Rothfuchs, N., Studart, A.R., 2012. Composites Reinforced in Three Dimensions by Using Low Magnetic Fields. Science 335, 199-204.

Espinosa, H.D., Rim, J.E., Barthelat, F., Buehler, M.J., 2009. Merger of structure and material in nacre and bone - Perspectives on de novo biomimetic materials. Prog. Mater. Sci. 54, 1059-1100.

Evans, A.G., Suo, Z., Wang, R.Z., Aksay, I.A., He, M.Y., Hutchinson, J.W., 2001. Model for the robust mechanical behavior of nacre. J. Mater. Res. 16, 2475-2484.

Katti, D.R., Katti, K.S., 2001. Modeling microarchitecture and mechanical behavior of nacre using 3D finite element techniques Part I Elastic properties. J. Mater. Sci. 36, 14111417.

Katti, K.S., Katti, D.R., Pradhan, S.M., Bhosle, A., 2005. Platelet interlocks are the key to toughness and strength in nacre. J. Mater. Res. 20, 1097-1100. 
Kolednik, O., Predan, J., Fischer, F.D., Fratzl, P., 2011. Bioinspired Design Criteria for Damage-Resistant Materials with Periodically Varying Microstructure. Adv. Funct. Mater. 21, 3634-3641.

Launey, M.E., Chen, P.Y., McKittrick, J., Ritchie, R.O., 2010. Mechanistic aspects of the fracture toughness of elk antler bone. Acta Biomater. 6, 1505-1514.

Launey, M.E., Munch, E., Alsem, D.H., Barth, H.B., Saiz, E., Tomsia, A.P., Ritchie, R.O., 2009. Designing highly toughened hybrid composites through nature-inspired hierarchical complexity. Acta Mater. 57, 2919-2932.

Launey, M.E., Ritchie, R.O., 2009. On the Fracture Toughness of Advanced Materials. Adv. Mater. 21, 2103-2110.

Le Ferrand, H., Bouville, F., Niebel, T.P., Studart, A.R., 2015. Magnetically assisted slip casting of bioinspired heterogeneous composites. Nat. Mater. 14, 1172-1179.

Libanori, R., Carnelli, D., Rothfuchs, N., Binelli, M.R., Zanini, M., Nicoleau, L., Feichtenschlager, B., Albrecht, G., Studart, A.R., 2016. Composites reinforced via mechanical interlocking of surface-roughened microplatelets within ductile and brittle matrices. Bioinspiration \& Biomimetics, accepted for publication.

Libanori, R., Erb, R.M., Studart, A.R., 2013. Mechanics of Platelet-Reinforced Composites Assembled Using Mechanical and Magnetic Stimuli. ACS Appl. Mater. Interfaces 5, 10794-10805.

Lopez, M.I., Martinez, P.E.M., Meyers, M.A., 2014. Organic interlamellar layers, mesolayers and mineral nanobridges: Contribution to strength in abalone (Haliotis rufescence) nacre. Acta Biomater. 10, 2056-2064.

Meyers, M.A., Chen, P.-Y., Lin, A.Y.-M., Seki, Y., 2008a. Biological materials: Structure and mechanical properties. Prog. Mater. Sci. 53, 1-206.

Meyers, M.A., Lim, C.T., Li, A., Hairul Nizam, B.R., Tan, E.P.S., Seki, Y., McKittrick, J., 2009. The role of organic intertile layer in abalone nacre. Mater. Sci. Eng. C 29, 2398-2410.

Meyers, M.A., Lin, A.Y.-M., Chen, P.-Y., Muyco, J., 2008b. Mechanical strength of abalone nacre: Role of the soft organic layer. J. Mech. Behav. Biomed. Mater. 1, 76-85.

Miserez, A., Rossoll, A., Mortensen, A., 2004. Investigation of crack-tip plasticity in high volume fraction particulate metal matrix composites. Eng. Fract. Mech. 71, 2385-2406.

Moës, N., Dolbow, J., Belytschko, T., 1999. A finite element method for crack growth without remeshing. Int. J. Numer. Meth. Engng. 46, 131-150.

Munch, E., Launey, M.E., Alsem, D.H., Saiz, E., Tomsia, A.P., Ritchie, R.O., 2008. Tough, BioInspired Hybrid Materials. Science 322, 1516-1520.

Naglieri, V., Gludovatz, B., Tomsia, A.P., Ritchie, R.O., 2015. Developing strength and toughness in bio-inspired silicon carbide hybrid materials containing a compliant phase. Acta Mater. 98, 141-151. 
Pimenta, S., Robinson, P., 2014. An analytical shear-lag model for composites with 'brickand-mortar' architecture considering non-linear matrix response and failure. Compos. Sci. Technol. 104, 111-124.

Podsiadlo, P., Kaushik, A.K., Arruda, E.M., Waas, A.M., Shim, B.S., Xu, J., Nandivada, H., Pumplin, B.G., Lahann, J., Ramamoorthy, A., Kotov, N.A., 2007. Ultrastrong and Stiff Layered Polymer Nanocomposites. Science 318, 80-83.

Preibisch, S., Saalfeld, S., Tomancak, P., 2009. Globally optimal stitching of tiled 3D microscopic image acquisitions. Bioinformatics 25, 1463-1465.

Rabiei, R., Bekah, S., Barthelat, F., 2010. Failure mode transition in nacre and bone-like materials. Acta Biomater. 6, 4081-4089.

Ritchie, R.0., 2011. The conflicts between strength and toughness. Nat. Mater. 10, 817822.

Sarikaya, M., 1994. An introduction to biomimetics: A structural viewpoint. Microsc. Res. Tech. 27, 360-375.

Schindelin, J., Arganda-Carreras, I., Frise, E., Kaynig, V., Longair, M., Pietzsch, T., Preibisch, S., Rueden, C., Saalfeld, S., Schmid, B., Tinevez, J.-Y., White, D.J., Hartenstein, V., Eliceiri, K., Tomancak, P., Cardona, A., 2012. Fiji: an open-source platform for biologicalimage analysis. Nat. Methods 9, 676-682.

Shao, Y., Zhao, H.P., Feng, X.Q., Gao, H.J., 2012. Discontinuous crack-bridging model for fracture toughness analysis of nacre. J. Mech. Phys. Solids 60, 1400-1419.

Smith, B.L., Schaffer, T.E., Viani, M., Thompson, J.B., Frederick, N.A., Kindt, J., Belcher, A., Stucky, G.D., Morse, D.E., Hansma, P.K., 1999. Molecular mechanistic origin of the toughness of natural adhesives, fibres and composites. Nature 399, 761-763.

Song, F., Soh, A.K., Bai, Y.L., 2003. Structural and mechanical properties of the organic matrix layers of nacre. Biomaterials 24, 3623-3631.

Sun, J., Bhushan, B., 2012. Hierarchical structure and mechanical properties of nacre: a review. RSC Advances 2, 7617-7632.

Tang, Z., Kotov, N.A., Magonov, S., Ozturk, B., 2003. Nanostructured artificial nacre. Nat. Mater. 2, 413-418.

Wang, R.Z., Suo, Z., Evans, A.G., Yao, N., Aksay, I.A., 2001. Deformation mechanisms in nacre. J. Mater. Res. 16, 2485-2493.

Wegst, U.G., Bai, H., Saiz, E., Tomsia, A.P., Ritchie, R.O., 2015. Bioinspired structural materials. Nat. Mater. 14, 23-36.

Xu, Z.-H., Yang, Y., Huang, Z., Li, X., 2011. Elastic modulus of biopolymer matrix in nacre measured using coupled atomic force microscopy bending and inverse finite element techniques. Mater. Sci. Eng. C 31, 1852-1856. 
Xu, Z.H., Li, X.D., 2011. Deformation Strengthening of Biopolymer in Nacre. Adv. Funct. Mater. 21, 3883-3888. 